1 Hacettepe Journal of Mathematics and Statistics

గ Volume 47 (1) (2018), 93-99

\title{
A graph associated to a fixed automorphism of a finite group
}

\author{
M. Mahtabi* and A. Erfanian ${ }^{\dagger \neq}$
}

\begin{abstract}
Let $G$ be a finite group and $A u t(G)$ be the group of automorphisms of $G$. We associate a graph to a group $G$ and fixed automorphism $\alpha$ of $G$ denoted by $\Gamma_{G}^{\alpha}$ as follows. The vertex set of $\Gamma_{G}^{\alpha}$ is $G \backslash Z^{\alpha}(G)$ and two vertices $x, g \in G \backslash Z^{\alpha}(G)$ are adjacent if $[g, x]_{\alpha} \neq 1$ or $[x, g]_{\alpha} \neq 1$, where $[g, x]_{\alpha}=g^{-1} x^{-1} g x^{\alpha}$ and $Z^{\alpha}(G)=\left\{x \in G \mid[g, x]_{\alpha}=1\right.$ for all $g \in G\}$. In this paper, we state some basic properties of the graph, like connectivity, diameter, girth and Hamiltonian. Moreover, planarity and 1-planarity are also investigated here.
\end{abstract}

Keywords: Automorphism group, diameter, independent set, dominating set, planer, outer planar.

2000 AMS Classification: Primary 05C25 ; Secondary 20P05.

Received: 02.09.2016 Accepted : 23.02.2017 Doi : 10.15672/HJMS.2017.456

\section{Introduction}

There are many papers on assigning a graph to a group and investigating the algebraic properties of the group through the associated graph. For instance, commuting graph [9], non-commuting graph [1], non-cyclic graph [3], non-normal graph [5], prime graph [12], power graph [7] and so on. In this paper, we are going to assign a new graph to a finite group $G$ and an automorphism $\alpha$ in the automorphism group $\operatorname{Aut}(G)$. Let $G$ be a finite group and $\alpha$ is an arbitrary but fixed element in $A u t(G)$. We define a graph denoted by $\Gamma_{G}^{\alpha}$ as an undirected simple graph with vertex set consisting all elements of $G \backslash Z^{\alpha}(G)$ and two distinct vertices $g, x \in G \backslash Z^{\alpha}(G)$ are adjacent whenever $[g, x]_{\alpha} \neq 1$ or $[x, g]_{\alpha} \neq 1$, where $[g, x]_{\alpha}=g^{-1} x^{-1} g x^{\alpha}$ and $Z^{\alpha}(G)$ is the set of all element $x$ in $G$ such that $[g, x]_{\alpha}=1$ for every $g \in G$. It is clear that if $\alpha$ is an identity automorphisms then $[x, g]_{\alpha}=[g, x]_{\alpha}$ and $\Gamma_{G}^{\alpha}$ is the know non-commuting graph. Moreover, if $G$ is an

\footnotetext{
${ }^{*}$ Department of Pure Mathematics, Ferdowsi University of Mashhad, Mashhad, Iran, Email: ma.mahtabi@stu.um.ac.ir

${ }^{\dagger}$ Department of Pure Mathematics and the Center of Excellence in Analysis on Algebraic Structures, Ferdowsi University of Mashhad, Mashhad, Iran, Email: erfanian@math.um.ac.ir

${ }^{\ddagger}$ Corresponding Author.
} 
abelian group, then $\Gamma_{G}^{\alpha}$ is a complete graph. Thus throughout the paper, we may always assume that $\alpha$ is non-identity automorphism and $G$ is a finite non-abelian group. Since we removed $Z^{\alpha}(G)$ from $G$, so it would imply that $\Gamma_{G}^{\alpha}$ has no isolated vertex.

In section 2, we investigate about the degree of vertices, diameter, girth and suitable condition for the graph to be Hamiltonian. Section 3 is devoted to a determination of some numerical invariants of the graph. Planarity, outer planarity and 1-planarity of the graph are also considered in section 4.

In the rest of this section, we remind some necessary definitions in graph theory. We remind that the girth of a graph is the length of a shortest cycle contained in the graph. The distance between two vertices in a graph is the number of edges in a shortest path connecting them. The diameter of a graph is the greatest distance between any pair of vertices. In graph theory, a dominating set for a graph $X$ with vertex set $V$ and edge set $E$ is a subset $\mathrm{D}$ of $\mathrm{V}$ such that every vertex not in $\mathrm{D}$ is adjacent to at least one member of $\mathrm{D}$. The domination number $\gamma(X)$ is the number of vertices in a smallest dominating set for $X$. An independent set or stable set is a set of vertices in a graph such that no two of which are adjacent. A planar graph is a graph that can be embedded in the plane, i.e., it can be drawn on the plane in such a way that its edges intersect only at their endpoints. In other words, it can be drawn in such a way that no edges crosses each other. Moreover, a 1-planar graph is a graph that can be drawn in the Euclidean plane in such a way that each edge has at most one crossing point, where it crosses a single additional edge. A Hamiltonian path is a path in a graph that visits each vertex exactly once a Hamiltonian cycle is a Hamiltonian path that is a cycle. A graph that contains a Hamiltonian cycle is called a Hamiltonian graph.

\section{Diameter and girth}

Let $G$ be a non-abelian finite group, and $\alpha$ an arbitrary but fixed element in $A u t(G)$. We denote $\alpha$-conjugacy class containing $x$ by $x_{\alpha}^{G}$ and it is clear that $x_{\alpha}^{G}=\left\{g^{-1} x g^{\alpha}: g \in\right.$ $G\}$. If $\left|x_{\alpha}^{G}\right|=1$, then $\alpha$ is nothing but conjugation by $x$. Moreover a subgroup $C_{G}^{\alpha}(x)=$ $\left\{g \in G \mid[x, g]_{\alpha}=1\right\}$ which satisfies $\left|x_{\alpha}^{G}\right|=\left[G: C_{G}^{\alpha}(x)\right]$. We have $\alpha$-center of the group $G$ as $Z^{\alpha}(G)=\bigcap_{x \in G} C_{G}^{\alpha}(x)=\left\{g \in G \mid[x, g]_{\alpha}=1\right.$ for all $\left.x \in G\right\}$. It is easy to show that $C_{G}^{\alpha}(x)$ is subgroup of $G$ and also $Z^{\alpha}(G)$ is normal subgroup of $G$. One can see that $Z^{\alpha}(G)=Z(G) \bigcap \operatorname{Fix}(\alpha)$ where $\operatorname{Fix}(\alpha)=\left\{x \in G: x^{\alpha}=x\right\}$. It is interesting to see that the number of generalized conjugacy classes is the number of ordinary conjugacy classes which are invariant under $\alpha$ and it is also equal to the number of irreducible characters which are invariant under $\alpha$ (see [4] for more details). By the above notations we may define subsets $R^{\alpha}(x)=\left\{g \in G \mid[g, x]_{\alpha}=1\right\}, T^{\alpha}(G)=\bigcap_{x \in G} R^{\alpha}(x)=\{g \in$ $G \mid[g, x]_{\alpha}=1$ for all $\left.x \in G\right\}$ of group $G, C_{G}^{\alpha}(S)=\left\{g \in G \mid[s, g]_{\alpha}=1\right.$ for all $\left.s \in S\right\}$ and $R_{G}^{\alpha}(S)=\left\{g \in G \mid[g, s]_{\alpha}=1\right.$ for all $\left.s \in S\right\}$, where $S$ is a subset of group $G$. First, let us remind the definition of graph as the following.

2.1. Definition. Let $G$ be a non-abelian finite group, and $\alpha$ an arbitrary but fixed element in $A u t(G)$. We define a graph, denoted by $\Gamma_{G}^{\alpha}$, such that vertex set of $\Gamma_{G}^{\alpha}$ is $G \backslash Z^{\alpha}(G)$ and two different vertices $x, g \in G \backslash Z^{\alpha}(G)$ are adjacent if $[g, x]_{\alpha} \neq 1$ or $[x, g]_{\alpha} \neq 1$.

In the following lemma, we give the degree of any vertex in $\Gamma_{G}^{\alpha}$.

2.2. Lemma. The degree of every vertex of the graph $\Gamma_{G}^{\alpha}$ is

(i) If $x=x^{\alpha}$ then $\operatorname{deg}(x)=|G|-\left|C_{G}(x) \bigcap C_{G}^{\alpha}(x)\right|$,

(ii) If $x \neq x^{\alpha}$ then $\operatorname{deg}(x)=|G|-\left|Z^{\alpha}(G)\right|-\left|R^{\alpha}(x) \bigcap C_{G}^{\alpha}(x)\right|-1$. 
Proof. For the degree of any vertex $x$ of the graph $\Gamma_{G}^{\alpha}$ we have two cases: $(i) x=x^{\alpha}$ and (ii) $x \neq x^{\alpha}$. In case $(i)$,

$$
\operatorname{deg}(x)=|G|-\left|Z^{\alpha}(G)\right|-\left|R^{\alpha}(x) \bigcap C_{G}^{\alpha}(x)\right|+\left|Z^{\alpha}(G) \bigcap R^{\alpha}(x)\right| .
$$

Furtheremore, if $x=x^{\alpha}$, then $R^{\alpha}(x)=C_{G}(x)$ and therefore $\operatorname{deg}(x)=|G|-\left|C_{G}(x) \bigcap C_{G}^{\alpha}(x)\right|$. In case $(i i)$ the degree of the vertex $x$ is

$$
|G|-\left|Z^{\alpha}(G)\right|-\left|R^{\alpha}(x) \bigcap C_{G}^{\alpha}(x)\right|+\left|Z^{\alpha}(G) \bigcap R^{\alpha}(x)\right|-1 .
$$

In this case $R^{\alpha}(x) \bigcap Z^{\alpha}(G)=\emptyset$ which implies that $\operatorname{deg}(x)=|G|-\left|Z^{\alpha}(G)\right|-\left|R^{\alpha}(x) \bigcap C_{G}^{\alpha}(x)\right|-$ 1.

2.3. Lemma. Let $G$ be a non-abelian finite group. Then the $\Gamma_{G}^{\alpha}$ is Hamiltonian graph.

Proof. Let $G$ be a non-abelian finite group. We know that if $x=x^{\alpha}$ then $\operatorname{deg}(x)=$ $|G|-\left|C_{G}(x) \bigcap C_{G}^{\alpha}(x)\right|$. Hence

$$
\begin{gathered}
\left|V\left(\Gamma_{G}^{\alpha}\right)\right|=|G|-\left|Z^{\alpha}(G)\right|<|G| \\
=2|G|-|G|<2|G|-2\left|C_{G}(x) \bigcap C_{G}^{\alpha}(x)\right| \\
=2\left(|G|-\left|C_{G}(x) \bigcap C_{G}^{\alpha}(x)\right|\right)=2 \operatorname{deg}(x) .
\end{gathered}
$$

Therefore $\operatorname{deg}(x)>\frac{\left|V\left(\Gamma_{G}^{\alpha}\right)\right|}{2}$.

If $x \neq x^{\alpha}$ then $\operatorname{deg}(x)=|G|-\left|Z^{\alpha}(G)\right|-\left|R^{\alpha}(x) \bigcap C_{G}^{\alpha}(x)\right|-1$. We have

$$
\begin{aligned}
& \left|V\left(\Gamma_{G}^{\alpha}\right)\right|=|G|-\left|Z^{\alpha}(G)\right|<|G|=2|G|-|G| \\
& \leqslant 2|G|-2\left(\left|Z^{\alpha}(G)\right|+\left|R^{\alpha}(x) \bigcap C_{G}^{\alpha}(x)\right|+1\right) .
\end{aligned}
$$

Thus $\operatorname{deg}(x)>\frac{\left|V\left(\Gamma_{G}^{\alpha}\right)\right|}{2}$ and by Dirac's Theorem [6], the graph $\Gamma_{G}^{\alpha}$ is Hamiltonian.

By the above lemma, we can see that $\Gamma_{G}^{\alpha}$ is always connected and also is not a star graph. Now in the following lemma, we determine of the diameter of $\Gamma_{G}^{\alpha}$.

2.4. Lemma. For any non-abelian group $G, \operatorname{diam}\left(\Gamma_{G}^{\alpha}\right)=2$.

Proof. First assume that there exists a vertex $x$ in $Z(G) \backslash \operatorname{Fix}(\alpha)$ and $y$ is an arbitrary vertex in $V\left(\Gamma_{G}^{\alpha}\right)$. Then $[y, x]_{\alpha}=y^{-1} x^{-1} y x^{\alpha}=x^{-1} x^{\alpha} \neq 1$, it implies that $x$ is adjacent to $y$ hence $d(x, y)=1$. Secondly, assume that there exists $x$ is an arbitrary vertex in $\operatorname{Fix}(\alpha) \backslash Z(G)$ and $y$ is an arbitrary vertex in $V\left(\Gamma_{G}^{\alpha}\right)$. Suppose that $y \neq y^{\alpha}$, then we have two cases, $(i) x$ and $y$ commute and $(i i) x$ does not commute with $y$. If case (i) occurs then $[x, y]_{\alpha}=[x, y] y^{-1} y^{\alpha} \neq 1$ thus $x$ is adjacent to $y$. If case (ii) occurs then $[y, x]_{\alpha}=y^{-1} x^{-1} y x^{\alpha}=[y, x] \neq 1$. It implies that $x$ is adjacent to $y$. Therefore $d(x, y)=1$. Assume that $y=y^{\alpha}$, since $\alpha$ is not identity automorphisms in $\operatorname{Aut}(G)$, so there exists $z \in G$ such that $z \neq z^{\alpha}$. Hence $z$ is a vertex in $V\left(\Gamma_{G}^{\alpha}\right)$. By the similar method, we can see that $z$ is adjacent to $x$ and $y$. It implies that $d(x, y)=2$. Finally assume that $x$ and $y$ are two non-adjacent vertices in $V\left(\Gamma_{G}^{\alpha}\right)$ such that $x, y \notin Z(G)$ and $x, y \notin \operatorname{Fix}(\alpha)$. We have $[x, x y]=[x, y] \neq 1$ hence $x y \notin Z(G)$. Thus $x y$ is a vertex in $\Gamma_{G}^{\alpha}$. We know that $[x, x y]_{\alpha}=[x, y]_{\alpha}[x, x]_{\alpha}^{y^{\alpha}}$. Since $x$ is not adjacent to $y$ so $[x, y]_{\alpha}=1$ and $[y, x]_{\alpha}=1$, hence $[x, x y]_{\alpha}=[x, x]_{\alpha}^{y^{\alpha}}$. Assume that $[x, x]_{\alpha}^{y^{\alpha}}=1$, then $[x, x]_{\alpha}=1$ it is a contradiction. Therefore $x$ is adjacent to $x y$. By the similar method we can show that $y$ is adjacent to $x y$. It implies that $d(x, y)=2$. The proof is now completed.

2.5. Lemma. Let $G$ be a non-abelian finite group. The girth of the graph $\Gamma_{G}^{\alpha}$ is at most 4. 
Proof. We have to show that $\Gamma_{G}^{\alpha}$ indeed has a cycle of length at most four. Firstly suppose that there exists a vertex $x$ in $Z(G) \backslash \operatorname{Fix}(\alpha)$. Let $y$ and $z$ be two arbitrary vertices in $V\left(\Gamma_{G}^{\alpha}\right)$. If $y$ is adjacent to $z$, then three elements $x, y, z$ induce a cycle of length 3 . If $y$ is not adjacent to $z$, then we can see that $y$ and $z$ are adjacent to $y z$. Hence elements $x, y, y z$ induce a cycle of length 3. Secondly, assume that $x$ is an arbitrary vertex in $\operatorname{Fix}(\alpha) \backslash Z(G)$ and $y, z$ are two vertex in $V\left(\Gamma_{G}^{\alpha}\right)$ such that $y \neq y^{\alpha}$ and $z \neq z^{\alpha}$. Then by Lemma 2.4. we have $x$ is adjacent to $y$ and $z$. If $y$ is adjacent to $z$, then elements $x, y, z$ induce a cycle of length 3. If $y$ is not adjacent to $z$, then we can see that $y$ and $z$ are adjacent to $y z$. Hence elements $x, y, z, y z$ induce a cycle of length 4 . Finally, $Z^{\alpha}(G)=Z(G)=\operatorname{Fix}(\alpha)$. Since $G$ is non-abelian group, there exists $x \in V\left(\Gamma_{G}^{\alpha}\right)$ such that $x^{2} \neq 1$, so $x^{-1}$ is vertex in $\Gamma_{G}^{\alpha}$ and therefore $x$ is adjacent to $x^{-1}$. For $y$ in $V\left(\Gamma_{G}^{\alpha}\right)$ that $y$ is adjacent to $x$, it is easy to show that $y$ is adjacent to $x^{-1}$. Thus elements $x, y, x^{-1}$ induce a cycle of length 3. Therefore the girth of $\Gamma_{G}^{\alpha}$ is at most 4 .

\section{Dominating set and independent set}

Suppose that $\left[G: Z^{\alpha}(G)\right]=r$. We know that $G$ can be partitioned into disjoint cosets of $Z^{\alpha}(G)$. Now, assume that $G \backslash Z^{\alpha}(G)=\bigcup_{i=1}^{r-1} g_{i} Z^{\alpha}(G)$.

3.1. Lemma. By the above notation let $g \in G \backslash Z^{\alpha}(G)$, then $g Z^{\alpha}(G)$ is an independent set in $\Gamma_{G}^{\alpha}$ if and only if $g \in \operatorname{Fix}(\alpha)$.

Proof. Assume that $x$ and $y$ two arbitrary elements in $g Z^{\alpha}(G)$ such that $x=g z_{1}$ and $y=g z_{2}$ where $z_{1}, z_{2} \in Z^{\alpha}(G)$. Let $x$ be not adjacent to $y$. We know that $x$ is not adjacent to $y$ if and only if $[x, y]_{\alpha}=1$ and $[y, x]_{\alpha}=1$. Hence $1=[x, y]_{\alpha}=\left[g z_{1}, g z_{2}\right]_{\alpha}=$ $[g, g]_{\alpha}\left[z_{2}, \alpha\right]$. It implies that $[g, g]_{\alpha}=[g, \alpha]=1$, thus $g \in \operatorname{Fix}(\alpha)$.

Let $A$ be an independent set of $\left\{g_{1}, g_{2}, \ldots, g_{r-1}\right\} \cap \operatorname{Fix}(\alpha)$ and $|A|=k, B=\bigcup_{a \in A} a Z^{\alpha}(G)$. By the above lemma, we can see that $\alpha\left(\Gamma_{G}^{\alpha}\right) \geq|B|=k\left|Z^{\alpha}(G)\right|$ where $\alpha\left(\Gamma_{G}^{\alpha}\right)$ is the independence number of $\Gamma_{G}^{\alpha}$. The following lemma states that every vertex of the coset $g Z^{\alpha}(G)$ is adjacent to every vertex of coset $x Z^{\alpha}(G)$ if and only if $g$ is adjacent to $x$, where $g$ and $x$ are in $G \backslash Z^{\alpha}(G)$.

3.2. Lemma. Let $g Z^{\alpha}(G)$ and $h Z^{\alpha}(G)$ be two arbitrary disjoint cosets of $G \backslash Z^{\alpha}(G)$, where $g, h \notin Z^{\alpha}(G)$. Then every element of $g Z^{\alpha}(G)$ is adjacent to every element of $h Z^{\alpha}(G)$ if and only if $g$ is adjacent to $h$.

Proof. Suppose that $x$ is an arbitrary element in $g Z^{\alpha}(G)$ and $y$ is an arbitrary element in $h Z^{\alpha}(G)$ such that $x=g z_{1}$ and $y=h z_{2}$ where $z_{1}, z_{2} \in Z^{\alpha}(G)$. Let $x$ is adjacent to $y$. We know that $x$ is adjacent to $y$ if and only if $[x, y]_{\alpha} \neq 1$ or $[y, x]_{\alpha} \neq 1$. Let $[x, y]_{\alpha} \neq 1$ then

$$
1 \neq[x, y]_{\alpha}=\left[g z_{1}, h z_{2}\right]_{\alpha}=[g, h]_{\alpha}\left[z_{2}, \alpha\right]=[g, h]_{\alpha} .
$$

Therefore $g$ is adjacent to $h$.

3.3. Lemma. Let $x$ be in $\operatorname{Fix}(\alpha)$. If $\{x\}$ is a dominating set of $\Gamma_{G}^{\alpha}$, then $Z^{\alpha}(G)=1$ and $x^{2}=1$.

Proof. Suppose on the contrary that $Z^{\alpha}(G) \neq 1$. Then every element of the set $x Z^{\alpha}(G)-$ $\{x\}$ is a vertex which is adjacent to $x$. This is a contradiction. Now, suppose that $x^{2} \neq 1$, then $x^{-1} \in V\left(\Gamma_{G}^{\alpha}\right)$. We have $\left[x, x^{-1}\right]_{\alpha}=1$, this is a contradiction. The proof is now complete.

3.4. Lemma. Let $G$ be a finite group. Then subset $S$ of $V\left(\Gamma_{G}^{\alpha}\right)$ is a dominating set of $\Gamma_{G}^{\alpha}$ if and only if $R^{\alpha}(S) \bigcap C_{G}^{\alpha}(S) \subseteq Z^{\alpha}(G) \cup S$. 
Proof. First assume that $S$ is a dominating set of $\Gamma_{G}^{\alpha}$ and $g \in G$. If $g \notin Z^{\alpha}(G) \cup S$, then $g \notin S$. Since $S$ is a dominating set, hence there exists $x \in S$ such that $g$ is adjacent to $x$. Thus $[x, g]_{\alpha} \neq 1$ or $[g, x]_{\alpha} \neq 1$. If $[x, g]_{\alpha} \neq 1$ then $g \notin C_{G}^{\alpha}(S)$. Also if $[g, x]_{\alpha} \neq 1$, then $g \notin R^{\alpha}(S)$. Therefore $R^{\alpha}(S) \bigcap C_{G}^{\alpha}(S) \subseteq Z^{\alpha}(G) \cup S$. Conversely, suppose that $R^{\alpha}(S) \bigcap C_{G}^{\alpha}(S) \subseteq Z^{\alpha}(G) \cup S$. If there exists $g \in G$ such that $g \notin Z^{\alpha}(G) \cup S$, then either $g \notin C_{G}^{\alpha}(S)$ or $g \in C_{G}^{\alpha}(S)$ but $g \notin R^{\alpha}(S)$. In each case, there exists $x \in S$ such that $[x, g]_{\alpha} \neq 1$ or $[g, x]_{\alpha} \neq 1$. It implies that $g$ is adjacent to $x$.

3.5. Lemma. Let $G$ be a non-abelian group such that $G=<X>$. Then $X \backslash Z^{\alpha}(G)$ is a dominating set of $\Gamma_{G}^{\alpha}$.

Proof. Suppose that $G$ is a non-abelian group such that $G=\langle X>$, then $Y=X \backslash$ $Z^{\alpha}(G) \neq \emptyset$. We show that $R^{\alpha}(Y) \bigcap C_{G}^{\alpha}(Y) \subseteq Z^{\alpha}(G) \cup Y$. Let $g \in R^{\alpha}(Y) \bigcap C_{G}^{\alpha}(Y)$, then $g \in C_{G}^{\alpha}(Y)=C_{G}^{\alpha}\left(X \backslash Z^{\alpha}(G)\right)$. Hence $g \in C_{G}^{\alpha}\left(<X>-Z^{\alpha}(G)\right)=C_{G}^{\alpha}\left(G-Z^{\alpha}(G)\right)$. Clearly $g \in C_{G}^{\alpha}\left(Z^{\alpha}(G)\right)$ and therefore $g \in Z^{\alpha}(G) \subseteq Z^{\alpha}(G) \cup Y$. Now, Lemma 3.4 implies that $X \backslash Z^{\alpha}(G)$ is a dominating set.

3.6. Lemma. Let $G$ be a non-abelian simple group and $\gamma\left(\Gamma_{G}^{\alpha}\right)$ be the dominating number of the graph $\Gamma_{G}^{\alpha}$. Then $\gamma\left(\Gamma_{G}^{\alpha}\right) \leqslant 2$.

Proof. Assume that $G$ is a non-abelian simple group, hence we can see that $G=\langle g, x>$. Since $G$ is a non-abelian simple group then $Z^{\alpha}(G)=1$, we set $X=\{g, x\}$. It is clear that $Z^{\alpha}(G) \bigcap X=\emptyset$, thus by Lemma 3.5, it is clear $X$ is a dominating set of $\Gamma_{G}^{\alpha}$.

\section{Planarity}

This section is devoted to a determination of planarity, outer planarity and 1-planar graph of the graph $\Gamma_{G}^{\alpha}$. We will show that, with exception of a few possible cases, $\Gamma_{G}^{\alpha}$ is not planar.

4.1. Lemma. If $\left|Z^{\alpha}(G)\right| \geq 3$, then $\Gamma_{G}^{\alpha}$ is not a planar graph.

Proof. Since $G$ is not abelian, so $\frac{G}{Z(G)}$ is not cyclic and so $[G: Z(G)] \geq 4$. Similarly $\left[G: Z^{\alpha}(G)\right] \geq 4$, because $Z^{\alpha}(G) \subseteq Z(G)$. Thus $G \backslash Z^{\alpha}(G)$ is the union of at least three distinct cosets of $Z^{\alpha}(G)$. Since $\Gamma_{G}^{\alpha}$ is connected, so there exists at least an edge between two cosets of $Z^{\alpha}(G)$. Now, by Lemma 3.2 and the assumption that $\left|Z^{\alpha}(G)\right| \geq 3$, we have a subgraph $K_{3,3}$. Therefore $\Gamma_{G}^{\alpha}$ is not planar.

4.2. Corollary. If $\left|Z^{\alpha}(G)\right| \neq 1$, then $\Gamma_{G}^{\alpha}$ is not an outer planar graph.

Proof. Let $\left|Z^{\alpha}(G)\right| \geq 2$, then by Lemma 4.1, we can see that $\Gamma_{G}^{\alpha}$ have a subgraph $K_{2,2}$. Hence $\Gamma_{G}^{\alpha}$ is not an outer planar graph.

We know that if $\Gamma_{G}^{\alpha}$ is a planar graph, then there exists a vertex $x$ such that $\operatorname{deg}(x) \leqslant 5$. Moreover if $\Gamma_{G}^{\alpha}$ is a 1 -planar graph, then there exists a vertex $y$ such that $\operatorname{deg}(y) \leqslant 7$ (see [8]). By this fact, we are going to state the following result.

4.3. Lemma. If $|G| \neq 6,8,10$ then $\Gamma_{G}^{\alpha}$ is not planar graph.

Proof. Suppose that $\Gamma_{G}^{\alpha}$ is a planar graph, then there exists a vertex $x$ in a $V\left(\Gamma_{G}^{\alpha}\right)$ such that $\operatorname{deg}(x) \leqslant 5$. First assume that $x=x^{\alpha}$, from Lemma 2.2 we see that $\operatorname{deg}(x)=$ $|G|-\left|C_{G}(x) \bigcap C_{G}^{\alpha}(x)\right|$. We have $\left|C_{G}(x) \bigcap C_{G}^{\alpha}(x)\right| \leqslant\left|C_{G}(x)\right|$. Thus $|G|-\left|C_{G}(x)\right| \leqslant$ 
$|G|-\left|C_{G}(x) \bigcap C_{G}^{\alpha}(x)\right| \leqslant 5$. Since $x$ is a vertex in $\Gamma_{G}^{\alpha}$, so $x \notin Z(G)$ and consequently $C_{G}(x) \varsubsetneqq G$. Therefore

$$
|G|-\frac{|G|}{2} \leqslant|G|-\left|C_{G}(x)\right| \leqslant|G|-\left|C_{G}(x) \bigcap C_{G}^{\alpha}(x)\right| \leqslant 5 .
$$

It implies that $|G| \leqslant 10$.

Second assume that $x \neq x^{\alpha}$ and $x \in Z(G)$, then $\operatorname{deg}(x)=|G|-\left|Z^{\alpha}(G)\right|-\left|R^{\alpha}(x) \bigcap C_{G}^{\alpha}(x)\right|-$ 1. Since $x \in Z(G)$ so $R^{\alpha}(x)=\emptyset$, hence $\operatorname{deg}(x)=|G|-\left|Z^{\alpha}(G)\right|-1$. We have $|G|-\frac{|G|}{2} \leqslant|G|-\left|Z^{\alpha}(G)\right| \leqslant 6$. Thus $|G| \leqslant 12$. Finally suppose that $x \neq x^{\alpha}$ and $x \notin Z(G)$ then $Z^{\alpha}(G) \leq Z(G) \supsetneqq C_{G}(x) \supsetneqq G$. Hence

$$
|G|-\frac{|G|}{4}-\left|C_{G}^{\alpha}(x)\right| \leqslant|G|-\left|Z^{\alpha}(G)\right|-\left|R^{\alpha}(x) \bigcap C_{G}^{\alpha}(x)\right| \leqslant 6 .
$$

It implies that $|G| \leqslant 24$. We know that the groups of orders $2,3,4,5,7,9,11,13,15$, $17,19,23$ are abelian groups. So associated graphs of the groups of orders $2,3,4,5,7,9$, $11,13,15,17,19,23$ are not planar graph. Moreover, if $\left|E\left(\Gamma_{G}^{\alpha}\right)\right| \leqslant 3\left|V\left(\Gamma_{G}^{\alpha}\right)\right|-6$, where $\left|E\left(\Gamma_{G}^{\alpha}\right)\right|$ is a number of edges in graph $\Gamma_{G}^{\alpha}$, then $\Gamma_{G}^{\alpha}$ is not planar (see [6, Corollary 9.5.2]. Hence by using the group theory package GAP [11], it is easy to see that graphs of the associated to groups of orders $12,14,16,18,20,21,22,24$ are not planar. It implies that if $|G| \neq 6,8,10$ then $\Gamma_{G}^{\alpha}$ is not planar graph.

By the above lemma, we can state that following corollary.

4.4. Corollary. If $|G| \neq 6,8,10,12,14,16$ then $\Gamma_{G}^{\alpha}$ is not 1 -planar graph.

Proof. If $\Gamma_{G}^{\alpha}$ is a 1 -planar graph, then there exists a vertex $x \operatorname{such}$ that $\operatorname{deg}(x) \leqslant 7$. Then we can see that if $x=x^{\alpha}$ then $|G| \leqslant 14$. If $x \neq x^{\alpha}$ and $x \in Z(G)$ then $|G| \leqslant 16$, and if $x \neq x^{\alpha}$ and $x \notin Z(G)$ then $|G| \leqslant 32$. Moreover, the groups of orders 2, 3, 4, 5, $7,9,11,13,15,17,19,23,25,29,31$ are abelian groups. So if $\Gamma_{G}^{\alpha}$ is a 1 -planar graph then $|G|$ is equal to $6,8,10,12,14,16,18,20,21,22,24,26,27,28,30,32$. Since every 1 -planar graph with $n$ vertices has at most $4 n-8$ edges, a straightforward computation shows that the associated graph of groups of orders 18,20,22, 24, 26, 27, 28, 30, 32 are not 1 -planar. The proof is now complete.

Acknowledgement. The authors would like to thank the referee for his/her careful reading and very useful comments and suggestions which improved the final version of this paper.

\section{References}

[1] A. Abdollahi, S. Akbari and H. R. Maimani, Non-commuting graph of a group, J. Algebra, 298 (2006), 468-492.

[2] A. Abdollahi, Engel graph associated with a group, J. Algebra, 318 (2007), 680-691.

[3] A. Abdollahi and A. Mohammadi Hassanabadi, Non-cyclic graph of a group, Comm. in Algebra, 35 (2007), 2057-2081.

[4] R. Barzegar and A. Erfanian, Nilpotency and solubility of groups relative to an automorphism, Caspian Journal of Mathematical Sciences, 4(2) (2015), 271-283.

[5] A. Erfanian, M. Farrokhi D.G. and B. Tolue, Non-normal graphs of finite groups, J. Algebra Appl., 12 (2013).

[6] J. A. Bondy and J. S. R. Murty, Graph Theory with Applications, Elsevier, (1977).

[7] P. J. Cameron and S. Ghosh, The power graph of a fnite group, Discrete Math, 311 (2011), $1220-1222$.

[8] I. Fabrici and T. Madaras, The structure of 1-planar graphs, Discrete Mathematics, 307 (2007), $854-865$. 
[9] A. Iranmanesh and A. Jafarzadeh, On the commuting graph associated with the symmetric and alternating groups, J. Algebra Appl., 7 (2008), 129-146.

[10] D. J. S. Robinson, A Course in the Theory of Groups, Springer-Verlag: New York-Heidelberg Berlin (1982).

[11] The GAP Group, GAP-Groups, Algorithms and Programming, Version 4.6.4, 2013 (http://www.gap-system.org/).

[12] J. S. Williams, Prime graph components of finite groups, J. Algebra, 69 (2) (1981), 487-513. 
